\title{
Information Chaos: An Adapted Framework Describing Citizens' Experiences with Information During COVID-19
}

\author{
Helen MONKMAN ${ }^{\mathrm{a}, 1}$, Andre W. KUSHNIRUK ${ }^{\mathrm{a}}$, Avi PARUSH ${ }^{\mathrm{b}}$, and Blake J. \\ LESSELROTH ${ }^{\mathrm{a}, \mathrm{c}}$ \\ ${ }^{a}$ School of Health Information Science, University of Victoria, Victoria, B.C., Canada \\ ${ }^{\mathrm{b}}$ Faculty of Industrial Engineering \& Management, Technion Israel Institute of \\ Technology, Haifa, Israel \\ ${ }^{\mathrm{c}}$ University of Oklahoma School of Community Medicine, Tulsa, Oklahoma, USA
}

\begin{abstract}
With the onset of the coronavirus pandemic, clinicians, public officials, and citizens alike struggled to stay abreast of the constant and evolving stream of information about the clinical manifestations of illness, epidemiology of the disease, and the public health response. In this paper, we adapted (i.e., added and modified elements) Beasley and colleagues' information chaos framework to understand the context of citizens' experiences with information during the COVID-19 pandemic. We will show how our adapted framework can be used to characterize information associated challenges observed during this time and the possible impact of information chaos on peoples' cognition and behaviours. Ultimately, we believe that research will benefit by adopting a more holistic perspective using the information chaos framework than strictly studying the independent factors in isolation.
\end{abstract}

Keywords. Consumer health informatics, information chaos, information overload, infodemic, COVID-19, coronavirus

\section{Introduction}

Humans living in the information age must process an overabundance of data. When the volume of data exceeds our processing capabilities, we face "information overload" [1] whereby excess information can adversely affect personal well-being, innovation, decision-making, and productivity [2]. In health care, information overload can have a pernicious effect upon population health outcomes [3]. Everyone struggled to stay abreast of the constant and evolving stream of medical information during the COVID19 pandemic [4]. Although information overload is arguably the most commonly known, there are many other kinds of information hazards that can also have consequences.

Beasley and colleagues introduced the concept of "information chaos" - a framework for characterizing information hazards primary care providers face that

${ }^{1}$ Helen Monkman, School of Health Information Science, University of Victoria, Victoria, British Columbia, Canada; E-mail: monkman@uvic.ca 
impair cognition and decision-making [5]. They argued that information chaos is "comprised of various combinations of information overload, information underload, information scatter, information conflict, and erroneous information" and that it can negatively affect physician performance and patient safety [5]. In this paper, we adapted Beasley's framework to describe citizens' experiences managing information during the COVID-19 pandemic and to illustrate how information chaos can influence behavioral outcomes (Figure 1). We selected Beasley's framework as the basis for this work because of its emphasis on the role of information itself, how it is processed, and the risks of information hazards. We also drew inspiration from other health information seeking models (e.g., [6]) because of their emphasis on the context, influencing variables, and the individual's agency. There are five concepts in this framework: (1) sources; (2) filter; (3) moderators; (4) hazards; and (5) behaviours. First, citizens received information from a variety of sources. Second, citizens filtered information for processing. Third, there were contextual factors (i.e., "moderators") mediating information processing. Fourth, there were multiple information hazards - including "infodemic", one we introduced. Finally, there were downstream behavioural outcomes. Next, we will apply this framework to illustrate how information chaos may have impeded cognition and public safety during the pandemic.

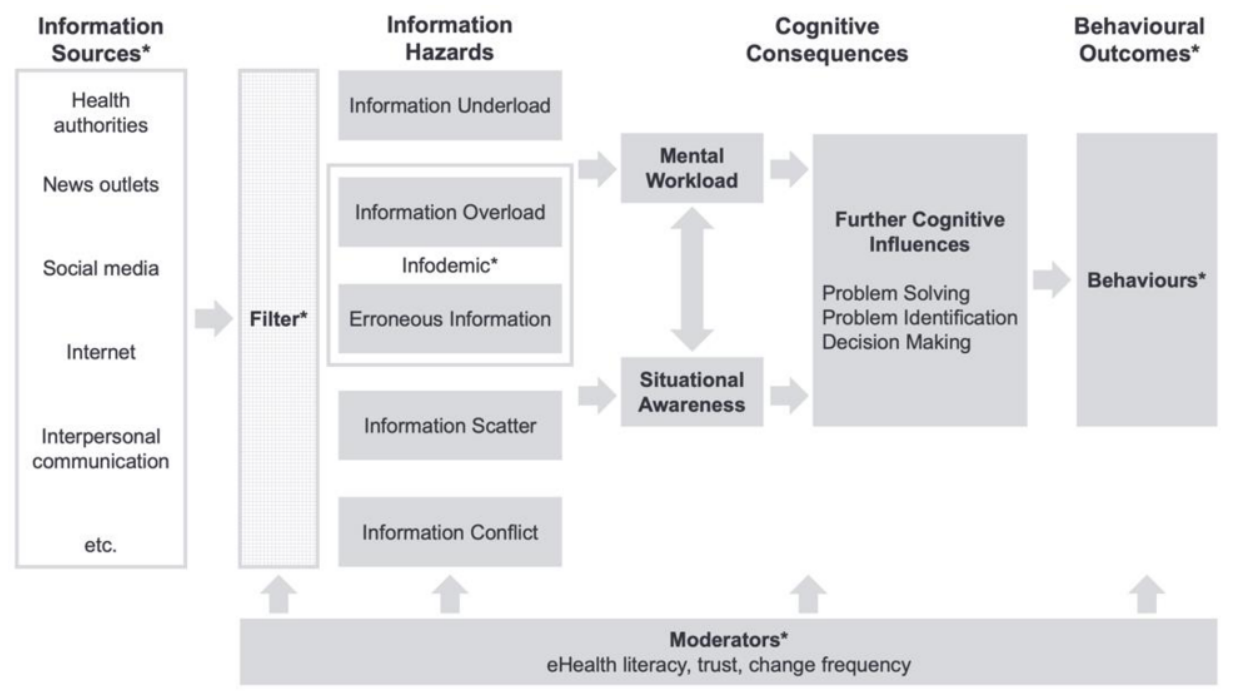

Figure 1. The information chaos framework for citizens' experiences with information during COVID-19, adapted from Beasley et. al's framework [5]. * = components added or significantly different from the Beasley et al.'s framework [5].

\section{Information Sources}

Citizens had to choose from many different information sources - some of questionable quality - during the pandemic. For example, citizens could use information provided by health authorities (e.g., national public health agencies, the World Health Organization [WHO]), public news outlets, social media (e.g., Twitter, Facebook, TikTok), the internet, and interpersonal communication (e.g., conversations, messaging apps). 
In our adapted framework, citizens had agency and participated in health information seeking, data consumption, information creation, and signal propagation. Individuals decide which health information to consume [6], so we included the concept of a filter in our framework to symbolize this decision-making process (see Figure 1).

\section{Moderators}

Moderators are external forces that influence internal system behaviour. Moderators acting on citizens during COVID-19 affected the entire information lifecycle, including the filter stage (see Figure 1). eHealth literacy, trust, and change frequency were likely the most influential moderators, but there are also other possible contextual and personal moderators (e.g., see [6]) as well. First, eHealth literacy is "the ability to seek, find, understand, and appraise health information from electronic sources and apply the knowledge gained to addressing or solving a health problem" [7]. Thus, eHealth literacy can influence the choice of information sources, comprehension, and decisions leading to behavioural outcomes (e.g., wearing a mask, physical distancing). Second, trust in information sources varies. For example, globally, $44 \%$ of people trust the news most of the time but only one in four people trust news sourced from social media [8]. If people do not trust a source, it is unlikely to pass their filter. Third, the rapid changes to information likely contributed to uncertainty, skepticism, and change fatigue.

\section{Information Hazards}

\subsection{Information Underload}

Information underload is "when necessary information is lacking" [5]. Citizens struggled with information underload throughout the pandemic; they did not have the information they needed at all times. For example, initial lack of information about disease virulence and mechanisms of transmission crippled early public safety measures. As the situation progressed, people wanted to learn about vaccine options, efficacy, and adverse effects.

\subsection{Information Overload}

Information overload occurs when data passing through the filter exceeds citizens' capabilities "to organize, synthesize, draw conclusions from, or act on" [5]. Quantity and relevancy can contribute to information overload. In a global health crisis, it is important to keep citizens abreast of an evolving situation with frequent updates. However, whether it was bulletins from work, broadcast media, social media, or print media, we struggled to keep up. People were more likely to report sentiments of information overload if they received or accessed COVID-19 information at a high frequency $[9,10]$ and also if the information source was broadcast media [9].

\subsection{Erroneous Information}

Erroneous information, or misinformation, can be unintentional or intentional (i.e., disinformation). Citizens undoubtedly encountered and suffered from erroneous 
information during the COVID-19 pandemic. We added an "infodemic" construct (see Figure 1) given that an infodemic is defined as "too much information, including false or misleading information, in digital and physical environments during a disease outbreak" [11]. Infodemics can compromise public trust in health authorities, leading to risky behaviours, and prolonging or potentiating outbreaks [11]. For example, erroneous information circulated about how the illness spread (e.g., only in cold weather), effective prophylactic measures (e.g., garlic), and treatments (e.g., spraying bleach on oneself) [12]. Social media users were more likely to report hearing misinformation about the pandemic [8]. Lies spread faster than the truth [13] and erroneous information on the internet can be nearly impossible to purge. Fortunately, some social media platforms are now taking action such as flagging false claims [14] or suspending users.

\subsection{Information Scatter}

Information scatter refers to the distribution and fragmentation of information in multiple locations [5]. Information about the pandemic was often fragmented amongst various information sources which contributed to the cognitive overhead. Beasley and colleagues' posited that users having difficulty finding the information they need, even within the same resource, is also a phenomenon associated with information scatter [5].

\subsection{Information Conflict}

Information conflict occurs when users are "unable to determine which data are correct" [5]. Because the science was evolving rapidly during the pandemic, recommendations were subject to change, which contributed to confusion [9]. For example, although health authorities did not clearly and consistently recommend donning masks initially, this recommendation later changed. Additionally, initially droplets were the suspected transmission mechanism, but later it was found to be aerosols [15].

\section{Cognitive Consequences and Behavioural Outcomes of Information Chaos}

As in Beasley and colleagues' [5], we applied a human factors engineering approach to model the consequences of information chaos and we extended this model to include behaviours . All hazards here were present throughout 2020 and varied as a function of information source and individual. These hazards undoubtedly influenced citizens' mental models, cognitive workload, situational awareness, emotional state, and eventual decision-making. Therefore, it follows that information chaos likely influenced peoples' behaviours (e.g., adhering to safety protocols, vaccine hesitancy).

\section{Discussion and Conclusion}

We adapted the information chaos framework and integrated health information seeking components to a contemporary public health context - COVID-19 - to illustrate the possible impact of information sources, consumer filters, information hazards, and moderating variables upon individual behaviours, epidemiologic patterns, and downstream public health outcomes. We also made several notable adaptions to this 
theoretical framework including the addition of information sources (i.e., where information comes from), a filter (i.e., whether people consume the information), and behavioural outcomes (i.e., what people do because of this information) as well as modifying the moderators (i.e., external contextual variables mediating information processing). A unified theoretical model to describe how information channels, hazards, and mediating forces influence behaviour, epidemiologic patterns, and population health could be a powerful tool for public officials in information campaigns and strategies.

While this model has face validity, future research is warranted to test its construct validity and predictive strength. We also need to investigate the interaction between variables and whether more integration with health information seeking models (e.g., [6]) is necessary. Finally, although researchers are exploring many of the factors described in our adapted framework independently, we believe a more holistic perspective of the potential interactions between variables may provide a more comprehensive representation of citizens' experiences during the pandemic.

\section{References}

[1] B.M. Gross, The managing of organizations; the administrative struggle, Free Press of Glencoe, New York, 1964.

[2] P. Hemp, Death by Information Overload, Harv. Bus. Rev. (2009). https://hbr.org/2009/09/death-byinformation-overload (accessed July 20, 2021).

[3] E.J. Huth, The information explosion., Bull. N. Y. Acad. Med. 65 (1989) 647-672.

[4] I. Klerings, A.S. Weinhandl, and K.J. Thaler, Information overload in healthcare: too much of a good thing?, Z. Für Evidenz Fortbild. Qual. Im Gesundheitswesen. 109 (2015) 285-290. doi:10.1016/j.zefq.2015.06.005.

[5] J.W. Beasley, T.B. Wetterneck, J. Temte, J.A. Lapin, P. Smith, A.J. Rivera-Rodriguez, and B.-T. Karsh, Information Chaos in Primary Care: Implications for Physician Performance and Patient Safety, J. Am. Board Fam. Med. 24 (2011) 745-751. doi:10.3122/jabfm.2011.06.100255.

[6] D.R. Longo, Understanding health information, communication, and information seeking of patients and consumers: a comprehensive and integrated model, Health Expect. Int. J. Public Particip. Health Care Health Policy. 8 (2005) 189-194. doi:10.1111/j.1369-7625.2005.00339.x.

[7] C.D. Norman, and H.A. Skinner, eHealth Literacy: Essential Skills for Consumer Health in a Networked World, J. Med. Internet Res. 8 (2006) e9. doi:10.2196/jmir.8.2.e9.

[8] N. Newman, R. Fletcher, A. Schulz, S. Andi, C.T. Robertson, and Nielsen, Reuters Institute Digital News Report 2021, 10th edition, Reuters Inst. Study Journal. (n.d.). https://reutersinstitute.politics.ox.ac.uk/sites/default/files/202106/Digital News Report 2021 FINAL.pdf (accessed July 9, 2021).

[9] M. Mohammed, A. Sha'aban, A.I. Jatau, I. Yunusa, A.M. Isa, A.S. Wada, K. Obamiro, H. Zainal, and B. Ibrahim, Assessment of COVID-19 Information Overload Among the General Public, J. Racial Ethn. Health Disparities. (2021) 1-9. doi:10.1007/s40615-020-00942-0.

[10] H. Hong, and H.J. Kim, Antecedents and Consequences of Information Overload in the COVID-19 Pandemic, Int. J. Environ. Res. Public. Health. 17 (2020) 9305. doi:10.3390/ijerph17249305.

[11] Infodemic, (n.d.). https://www.who.int/westernpacific/health-topics/infodemic (accessed July 6, 2021).

[12] M. Cha, C. Cha, K. Singh, G. Lima, Y.-Y. Ahn, J. Kulshrestha, and O. Varol, Prevalence of Misinformation and Factchecks on the COVID-19 Pandemic in 35 Countries: Observational Infodemiology Study, JMIR Hum. Factors. 8 (2021) e23279. doi:10.2196/23279.

[13] S. Vosoughi, D. Roy, and S. Aral, The spread of true and false news online, Science. 359 (2018) 11461151. doi: $10.1126 /$ science.aap9559.

[14] C. Lanius, R. Weber, and W.I. MacKenzie, Use of bot and content flags to limit the spread of misinformation among social networks: a behavior and attitude survey, Soc. Netw. Anal. Min. 11 (2021) 32. doi:10.1007/s13278-021-00739-x.

[15] V.S. Salian, J.A. Wright, P.T. Vedell, S. Nair, C. Li, M. Kandimalla, X. Tang, E.M. Carmona Porquera, K.R. Kalari, and K.K. Kandimalla, COVID-19 Transmission, Current Treatment, and Future Therapeutic Strategies, Mol. Pharm. (2021) acs.molpharmaceut.0c00608. doi:10.1021/acs.molpharmaceut.0c00608. 